\title{
ELEVEN WEEKS OF STRENGTH TRAINING DECREASED THE EXPRESSION OF IMMUNE-RELATED GENES IN OLDER SUBJECTS INDEPENDENT OF PROTEIN SUPPLEMENT TYPE; A RANDOMIZED CONTROLLED TRIAL
}

\author{
G.O. Gjevestad ${ }^{1,2}$, H. Hamarsland ${ }^{3}$, T. Raastad ${ }^{3}$, J.J. Christensen ${ }^{1,4}$, A.S. Biong ${ }^{2}$, S.M. Ulven ${ }^{1}$, K.B. Holven H., $^{1,5}$
}

\begin{abstract}
Objective: To investigate the effects of eleven weeks of strength training combined with two isocaloric protein supplements on mRNA expression levels in skeletal muscle and peripheral blood mononuclear blood cells (PBMCs). Design: A double blind randomized controlled study. Setting: The Norwegian School of Sports Sciences, Norway. Participants: Untrained, but otherwise healthy, men and women $(n=20, \geq 70$ yrs). Intervention: Participants were randomly allocated to receive either milk protein or a native whey protein supplement (20 g protein, morning and afternoon) combined with a standardized strength training protocol (6-10 RM, 1-3 sets, 3 times/week) for eleven weeks. Measurements: The mRNA expression levels of immunerelated genes were measured before and after the intervention period, using RT-qPCR. Cytokines were measured using ELISA. Results: PBMC mRNA expression of interleukin (IL) 6, IL8, chemokine (C-C motif) ligand (CCL) 3, and nuclear receptor subfamily (NR) 1 group $\mathrm{H}(\mathrm{H})$ member 3 decreased significantly after the intervention period, whereas the mRNA expression of tolllike receptor (TLR2) increased. In skeletal muscle, the mRNA expression of peroxisome proliferator-activated receptor gamma coactivator 1 alpha (PPARGC1A) and PPARGC1B decreased significantly, whereas the mRNA expression of CCL2, CCL5, TLR2, TLR4 and hypoxia inducible factor 1 alpha subunit (HIF1A) increased significantly after the intervention. We found no significant differences in circulating C-reactive protein and IL6 after the intervention period. The consumption of whey and milk proteins had similar effects on mRNA expression levels after strength training in skeletal muscle as well as PBMCs. Conclusion: Eleven weeks of strength training and protein supplementation reduced the PBMC expression levels of genes involved in the immune system as well as in metabolism, underlining the close interaction between these processes. The upregulation of other immune-related genes observed in PBMCs as well as in skeletal muscle needs further investigations, but may be related to protein supplementation and training adaptations. Different protein supplementation (milk or native whey) did not differentially modulate mRNA expression after the intervention period.
\end{abstract}

Key words: PBMC, skeletal muscle, mRNA, resistance training, cytokines.

Abbreviations: BCAA, branched chain amino acids; CCL, chemokine (C-C motif) ligand; RCT, reverse cholesterol transport; CRP, C-reactive protein; CXCL, chemokine (C-X-C motif) ligand; E \%, energy percent; HIF1A, hypoxia-inducible factor 1-alpha; HMBS, hydroxymethylbilane synthase; IL, interleukin; IL1RN, interleukin 1 receptor antagonist; IMVC, isometric maximum voluntary contraction; IPO8, importin 8; NR4A2; NF- $\kappa \mathrm{B}$, nuclear factor kappa-light-chain-enhancer of activated B cells; nuclear receptor subfamily 4, group A, member 2; NR4A3; nuclear receptor subfamily 4, group A, member 3; NR1H3, Nuclear Receptor Subfamily 1 Group H Member 3; PBMCs, peripheral blood mononuclear cells; PPARGC1A, peroxisome proliferator-activated receptor gamma coactivator 1-alpha; PPARGC1B, peroxisome proliferator-activated receptor gamma coactivator 1-beta; RCT, reverse cholesterol transport; RM, repetition maximum; RT-qPCR, real-time quantitative polymerase chain reaction; TBP, TATA box binding protein; TG, triglycerides, TLDA, TaqMan Low-Density array; TLR, toll-like receptor; TNF, tumor necrosis factor alpha.

1. Department of Nutrition, Institute of Basic Medical Sciences, P.O. Box 1046, Blindern, 0317 University of Oslo, Norway; 2. TINE SA, Centre for Research and Development, P.O. Box 7, Kalbakken, 0902 Oslo, Norway; 3. Department of Physical Performance, Norwegian School of Sport Sciences, P.B. 4104 U.S., 0806 Oslo, Norway; 4. The Lipid Clinic, Oslo University Hospital Rikshospitalet, P.O. Box 4950 Nydalen, 0424 Oslo, Norway; 5. National Advisory Unit on Familial Hypercholesterolemia, Department of Endocrinology, Morbid Obesity and Preventive Medicine, Oslo University Hospital, P.O. Box 4950 Nydalen, 0424 Oslo, Norway.

Corresponding Author: Kirsten B. Holven, Department of Nutrition, Institute of Basic Medical Sciences, P.O. Box 1046, Blindern, 0317 University of Oslo, Norway. k.b.holven@medisin.uio.no

\section{Introduction}

Diet and exercise are two of the most important lifestyle factors influencing healthy aging. Both are able to induce changes in metabolism and immune function $(1,2)$. They are therefore important targets for lifestyle interventions aiming at preventing the development of age-related diseases, including cardiovascular diseases, diabetes type 2, obesity (3) and sarcopenia (4).

Regular physical activity improves several of the 
underlying metabolic processes that are associated with adverse health effects and age-related diseases, such as insulin sensitivity, plasma triglycerides, blood pressure, and endothelial function (5). Regular strength training promotes muscle hypertrophy mainly, being particularly important in the prevention of age-associated loss of muscle mass and function associated with sarcopenia (6). Cross-sectional and large-scale cohort-studies have consistently shown an inverse association between physical activity and circulating markers of low-grade chronic inflammation (7). This anti-inflammatory effect is suggested to be one of the mechanisms underlying the protective effects observed on the development of chronic metabolic diseases by regular exercise (5), including sarcopenia (8). Because older adults often have a higher baseline level of circulating immune-related markers (9), long term regular exercise has been suggested as a tool to ameliorate the inflammatory status of older adults (10).

Components of the diet may exert anti-inflammatory effects and protect against chronic low-grade inflammation (2). In line with this, some epidemiological findings indicate that low-fat dairy products may reduce circulating levels of immune-related markers (11). However, others have found no association between intake of dairy products and circulating levels of immune-related markers, and conclusions from intervention studies have been conflicting (12). Although possible mechanisms underlying a potential anti-inflammatory effect of dairy products remains to be elucidated, several components in milk have been suggested to extert the anti-inflammatory effects, among them whey proteins (13).

The aims of the present study were to investigate the effects of eleven weeks of strength training combined with two isocaloric protein supplements $(20 \mathrm{~g}$ protein morning and afternoon) on mRNA expression levels in skeletal muscle and peripheral blood mononuclear blood cells (PBMCs) in healthy older adults ( $>70 \mathrm{yrs})$.

\section{Materials and methods}

\section{Study population and experimental design}

Twenty-four older ( $\geq 70 \mathrm{yrs}$ ) untrained men and women were recruited to an eleven week lasting double-blind, randomized controlled study, which was conducted from August 2014 to December 2014 at The Norwegian School of Sport Sciences, Norway. All participants provided written informed consent, and we conducted the study according to the guidelines laid down in the Declaration of Helsinki. The Regional Committees for Medical and Health Research Ethics, Health Region South East, Norway, provided approval for all planned procedures involving human subjects $(2014 / 834)$.

Subjects were randomized into one of two groups, receiving dairy supplements based on either native whey protein or regular milk protein for eleven weeks, on top of a high-load strength training regime. All tests were performed before and after the 11-week period. From the day before the test days, subjects followed a standardized diet based on body weight. In the morning of the test day, subjects were served a standardized breakfast, consisting of oatmeal, water, rapeseed oil and sugar (50 E \% from carbohydrate, $8 \mathrm{E} \%$ from protein and $42 \mathrm{E} \%$ from fat). All subjects finished the breakfast within 20 minutes. PBMCs and skeletal muscle samples were collected $90 \mathrm{~min}$ after breakfast was served. We used Dual energy X-ray absorptiometry (Lunar iDXA, GE Healthcare, Buckinghamshire, United Kingdom) and enCORE Software (version 14.10.022, GE Lunar) to determine fat mass. Android fat mass was estimated based on algorithms in the enCORE Software. All subjects reported to be healthy, but one participant (4\%) had prescribed anticoagulants and six participants $(25 \%)$ took statins. Four subjects did not complete the study due to disease (two participants) or hurting joints (two participants).

\section{Protein supplements}

TINE SA (Oslo, Norway) provided the supplements. The products contained $20 \mathrm{~g}$ of protein $(27 \mathrm{E} \%), 39 \mathrm{~g}$ carbohydrates $(52 \mathrm{E} \%)$ and $7 \mathrm{~g}$ fat $(21 \mathrm{E} \%)$, providing approximately $300 \mathrm{kcal}$ per serving. All subjects received $2 \times 20 \mathrm{~g}$ protein/day. The protein source was either native whey protein or regular milk protein (approximately $80 \%$ casein and $20 \%$ whey). Native whey proteins differ from regular whey proteins by the production method (produced at low temperatures to avoid extensive denaturation of the protein) and the composition of amino acids (e.g. higher amount of leucine). The participants prepared one serving in the morning and one in the evening, except at training days, where the training instructors provided the evening dose following the exercise sessions. The powder was dissolved in approximately $500 \mathrm{ml}$ water. The producer delivered supplements in identical and coded packaging to ensure blinding of both the providers and the participants. The producer was responsible for coding of the products, and the coding was not revealed until the intervention was completed. All products had the same flavor, color and appearance.

\section{Training protocol}

The training program was standardized in terms of exercises performed (hammer squat, leg press, knee extensions, calf raise, chest press, seated rowing, close grip pull down, shoulder press, back extensions and crunches), the number of sets (6-12 repetition maximum, $\mathrm{RM})$ and the number of repetitions (1-3 sets in each exercise). The exercise program was performed 3 days per week for eleven weeks (Figure 1), and all training sessions were supervised by trainers. The load in each exercise 
was individualized and adjusted each training session by the trainer to make sure that participants always exercised at the intended training load. Participants completed the last exercise session at least three days before the last biopsy was taken.

\section{Sampling and sample preparation}

We collected venous blood samples in BD Vacutainer ${ }^{\circledR}$ $\mathrm{CPT}^{\mathrm{TM}}$ cell preparation tubes with sodium heparin (Becton Dickinson, NJ, USA) and in silica gel tubes (Becton Dickenson Vacutainer Systems, Plymouth, UK). Within two hours of blood collection, PBMCs were isolated according to the manufacturer's instructions (Becton Dickinson, NJ, USA). Serum samples were centrifuged (1500 g, $15 \mathrm{~min}$ at RT) after being left on the bench top for at least $30 \mathrm{~min}$. Blood plasma samples were collected with pipettes immediately after being collected (lithium heparin tubes from Vacuette, Greiner Bio One, Austria) and centrifuged $\left(1300 \mathrm{~g}, 10 \mathrm{~min}\right.$ at $\left.4^{\circ} \mathrm{C}\right)$. All samples were stored at $-80^{\circ} \mathrm{C}$ until further analysis.

Muscle biopsies from the $m$. vastus lateralis were obtained at the same time points as the blood samples, using a modified Bergstrom technique (14), immediately cleaned from blood and connective tissue in physiological salt water at $4^{\circ} \mathrm{C}$, immersed into RNAlater ${ }^{\circledR}$ solution (Ambion, TX, USA) and stored overnight at $4{ }^{\circ} \mathrm{C}$. The following day, the biopsies were transferred and stored at $-80{ }^{\circ} \mathrm{C}$ until further analysis. Biopsies were taken from the same leg before and after the intervention.

\section{Isolation of RNA and synthesis of cDNA}

Skeletal muscle samples were ruptured using a CryoGrinder System (Ops Diognostics, NJ, USA), followed by homogenization in Quizol (QIAGEN $\mathrm{GmbH}$, Germany) and addition of chloroform. After centrifugation, the upper phase was transferred to a fresh tube and ethanol added. The procedure of RNA isolation was carried out using the QiaCube instrument (QIAGEN GmbH, Germany) following the miRNease Mini Kit protocol (QIAGEN). We isolated RNA from PBMCs in accordance with the RNeasy Mini Kit protocol using qiashredder and DNase digest (QIAGEN GmbH, Germany) using the QiaCube. High-quality RNA from both PBMCs and skeletal muscle samples was eluted in $30 \mu \mathrm{l}$ of RNase free water and frozen at $-80{ }^{\circ} \mathrm{C}$ until further analysis. RNA quantity was measured using NanoDrop-1000 (NanoDrop Technologies, Inc., DE, USA), and RNA quality was checked with Aglient 2100 Bioanalyzer (Agilent Technologies, Inc., CA, USA). All samples included in further analysis had a RIN-value above 5.5. PBMC samples from one person were not taken due to a misunderstanding in the laboratory, whereas mRNA from skeletal muscle was lost from six participants due to low RIN-values. In addition, we excluded some of the mRNA transcripts from our final analysis due to abnormal multicomponent plots.

RNA samples were transcribed into cDNA (500 ng) using the cDNA kit from Applied Biosystems (Applied Biosystems, UK) and in accordance with the protocol provided. Samples were stored at $-20{ }^{\circ} \mathrm{C}$ for further analysis.

\section{RNA analysis by real-time qPCR}

We measured mRNA levels of 48 genes (Additional file 1) using TaqMan Low-Density array (TLDA) cards from Applied Biosystems (UK). The TLDA cards were used on a 7900 HT Applied Biosystems RT-qPCR machine (Applied Biosystems, UK). The Ct-values were analysed using SDS 2.4 (Applied Biosystems, UK), and further transferred to ExpressionSuite Sofware v1.0.3 (Applied Biosystems, UK). For PBMCs, we normalised the Ct-values to TATA box binding protein (TBP) and hydroxymethylbilane synthase (HMBS) mRNA transcripts, whereas for skeletal muscle, we normalized to TBP and importin 8 (IPO8). Fold changes in mRNA transcripts from baseline to end of study were calculated, using the $2^{-\Delta \Delta C t}$-method (15).

The selection of genes were based on a thorough literature search investigating the effect of training on gene expression in PBMCs (16). We performed a similar research for skeletal muscle (17). Moreover, the genes selected in the present study were based on previously published studies where the effects of dairy products on markers of chronic low-grade inflammation were described (18).

\section{Cytokine measurements and routine analysis}

The serum level of IL6 was measured with highsensitive Quantikine ELISA (R\&D Systems Inc., MN, USA), whereas IL8 and CCL3 were measured using Quantikine ELISA (R\&D Systems Inc.), both in accordance with the protocols provided. We measured all samples in duplicates. Serum levels of glucose, triglycerides, cholesterol and the plasma level of C-reactive protein (CRP) were analyzed by an accredited laboratory (Fürst Medical Laboratory, Oslo; Norway).

\section{Statistics}

Power calculation was performed for the primary outcome of the study, which was to study the effects of consuming native whey or milk protein on muscle mass and strength. We also considered this number of participants to be relevant with respect to changes in inflammatory markers. In addition, the number of participants included in the present study is in line with other studies exploring the relationships between exercise and gene expression. All data were checked for normality. Subjects with levels of CRP above $>10 \mathrm{mg} / \mathrm{L}$ at baseline $(n=1)$ or at end of study $(n=1)$ were excluded 
from the gene expression analysis as such levels may indicate an ongoing acute inflammation, not reflecting the intervention. For non-parametric data, we used the Mann-Whitney-test for independent measurements and the Wilcoxon signed-rank for repeated, paired measurements. For parametric data, Independent samples t-test was used for independent measurements and Paired t-test for paired measurements. The Spearman correlation test was used to reveal possible correlations between the change in android fat mass and mRNA expression levels of selected transcripts. Due to an explorative study design, correction for multiple testing was not performed. We considered a p-value of $<0.05$ statistically significant. SPSS statistical software, version 22 from Microsoft (SPSS, Inc., CA, USA), was used for statistical calculations and GraphPad Prism 5 (GraphPad Software, Inc., CA, USA) for generating figures.

\section{Results}

Participants $(n=20$, mean $\pm S D=73.6 \pm 2.8$ yrs $)$ included in the present study were similar in body mass, fat mass, BMI and blood parameters at baseline. The proportion of male to female was $12 / 8$. We observed a significant change in body mass and BMI from baseline to end of intervention, but not in fat mass or android fat mass (Table 1). None of the anthropometric characteristics or blood parameters changed significantly from baseline to end of study between the native whey and the milk group (data not shown).

Table 1

Anthropometric parameters at baseline and after the intervention

\begin{tabular}{lcccc}
\hline & \multicolumn{3}{c}{ Participants (n=20) } & \\
& $\begin{array}{c}\text { Mean, } \\
\text { baseline } \\
( \pm \text { SD) }\end{array}$ & $\begin{array}{c}\text { Mean, end } \\
( \pm \text { SD) }\end{array}$ & $\begin{array}{c}\Delta \text { mean } \\
( \pm \text { SD) }\end{array}$ & p-value $^{1}$ \\
\hline Body mass (kg) & $76.7 \pm 14.7$ & $79.2 \pm 14.7$ & $2.6 \pm 1.5$ & $>0.001$ \\
Fat mass (kg) & $23.7 \pm 8.2$ & $24.0 \pm 8.1$ & $0.3 \pm 1.0$ & 0.17 \\
Android fat mass (\%) & $35.4 \pm 10.5$ & $35.2 \pm 9.9$ & $-0.2 \pm 2.8$ & 0.68 \\
BMI (kg/m2) & $25.5 \pm 4.1$ & $26.3 \pm 4.1$ & $0.9 \pm 0.5$ & $>0.001$ \\
Glucose (mmol/L)2 & $5.4 \pm 0.7$ & $5.6 \pm 0.7$ & $0.17 \pm 0.57$ & 0.21 \\
Triglycerides (mmol/L) & $1.2 \pm 0.5$ & $1.3 \pm 0.6$ & $0.07 \pm 0.43$ & 0.46 \\
Cholesterol (mmol/L) ${ }^{2}$ & $5.4 \pm 1.1$ & $5.4 \pm 1.0$ & $-0.04 \pm 0.59$ & 0.79 \\
LDL (mmol/L) & $3.6 \pm 1.0$ & $3.6 \pm 0.9$ & $-0.02 \pm 0.62$ & 0.91 \\
HDL (mmol/L)2 & $1.6 \pm 0.3$ & $1.5 \pm 0.3$ & $-0.05 \pm 0.14$ & 0.13 \\
\hline
\end{tabular}

1. Calculated by Paired sample t-test; $2 . \mathrm{n}=19$; Abbreviations used in table; $\Delta$, delta; HDL, high-density cholesterol; LDL, low-density cholesterol

\section{Adherence to the strength training and protein supplementation}

Participants attended an average of $33.0 \pm 0.9$ and 32.5 \pm 1.2 exercise sessions (of totally 33 exercise sessions) in the native whey and milk group, respectively. We logged adherence to the supplementation regime at each training session, which resulted in a mean self-reported compliance of $99 \%$.

\section{Effects of strength training and protein supplementation on gene expression}

Protein supplements based on milk protein or native whey protein did not significantly alter mRNA expression levels after eleven weeks of strength training neither in PBMCs nor in skeletal muscle. When merging the two groups, PBMC mRNA expression levels of interleukin (IL) 6, IL8, chemokine (C-C motif) ligand (CCL) 3 and nuclear receptor subfamily 1 , group $A$, member 3 (NR1H3, also known as LXR) were significantly reduced after the intervention period, whereas the mRNA expression level of toll-like receptor (TLR) 2 increased (Figure 2). In skeletal muscle, the mRNA expression levels of CCL2, CCL5, TLR2, TLR4, IL8 and hypoxia-inducible factor 1-alpha (HIF1A) significantly increased after the intervention (Figure 3 and 4), whereas peroxisome proliferator-activated receptor gamma coactivator -alpha (PPARCG1A) and PPARCG1B decreased significantly (Figure 4). We also observed significant changes in the expression of some genes related to lipid metabolism, both in PMBCs and skeletal muscle, as shown in Additional file 2 and 3.

\section{Circulating immune-related markers}

No differences were observed from baseline to after the intervention in the serum level of IL6 or the plasma level of CRP (Table 2). Serum levels of IL8 and CCL3 were also measured, but were not detectable (not shown).

Table 2

Inflammatory markers at baseline and after the intervention

\begin{tabular}{lcccc}
\hline & \multicolumn{3}{c}{ Participants $(\mathbf{n}=\mathbf{1 7})$} \\
& $\begin{array}{c}\text { Median, baseline } \\
( \pm \mathrm{SD})\end{array}$ & $\begin{array}{c}\text { Median, } \\
\text { end }( \pm \mathrm{SD})\end{array}$ & $\begin{array}{c}\Delta \text { median } \\
( \pm \mathrm{SD})\end{array}$ & $\begin{array}{c}\text { p-value }^{\mathbf{1}} \\
\text { (baseline-end) }^{-}\end{array}$ \\
\hline $\mathrm{CRP}(\mathrm{mg} / \mathrm{L})^{2}$ & $1.0 \pm 1.8$ & $1.0 \pm 1.2$ & $0.0 \pm 2.2$ & 0.44 \\
$\mathrm{IL6}(\mathrm{pg} / \mathrm{L})$ & $1.6 \pm 0.4$ & $1.6 \pm 0.9$ & $-0.03 \pm 0.7$ & 0.19 \\
\hline
\end{tabular}

1. Calculated by Wilcoxon signed rank test; 2 . $\mathrm{n}=14$; Abbreviations used in table; $\mathrm{CRP}, \mathrm{C}$-reactive protein; $\Delta$, delta; IL, interleukin.

\section{Correlations between android fat mass and immune-related genes and circulating markers}

No correlations between changes in android fat mass and changes in mRNA expression levels of IL6, IL8 and CCL3 in PBMCs or circulating levels of IL6 and CRP were observed from baseline to end of intervention. 
Figure 1

Study design and timeline for sampling during the intervention study

Baseline 20 g protein $\times 2 /$ day
High-load strength training
3 days/week, 6-12 RM, 1-3 sets

\section{Figure 2}

mRNA expression levels in PBMCs before and after the intervention

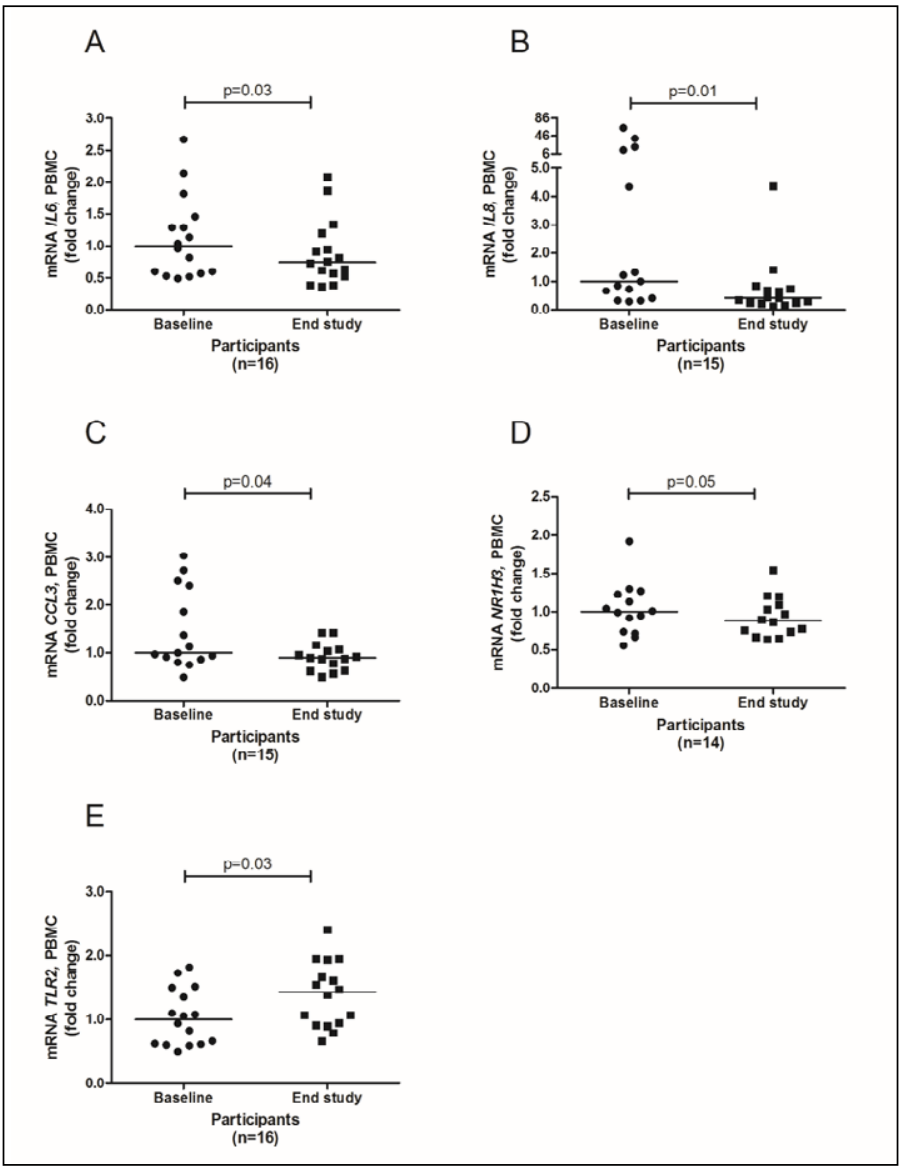

Expression levels of IL6 [A], IL8 [B], CCL3 [C], NR1H3 [D] and TLR2 [E] from baseline to 11 weeks of strength exercise. Values are expressed as fold changes, and the vertical lines represent median values. The $p$-values were calculated by Wilcoxon signed-rank test and indicate changes from baseline til end of study. $\mathrm{n}=$ $14[\mathrm{D}], \mathrm{n}=15[\mathrm{~B}, \mathrm{C}]$ and $\mathrm{n}=16[\mathrm{~A}, \mathrm{G}]$.

\section{Discussion}

We observed that 11 weeks of high-load strength training combined with protein supplementation decreased mRNA expression levels of several immunerelated genes in PBMCs, potentially having beneficial effects on systemic low-grade inflammation. On the other hand, immune-related mRNA transcripts were both up- and downregulated in skeletal muscle, probably reflecting muscle regeneration and adaptation. Native whey and milk proteins did not differentially alter mRNA expression levels, neither in PBMCs nor in skeletal muscle after strength training. No effects were observed on circulating levels of IL6 or CRP.

\section{Figure 3}

mRNA expression levels in skeletal muscle before and after the intervention

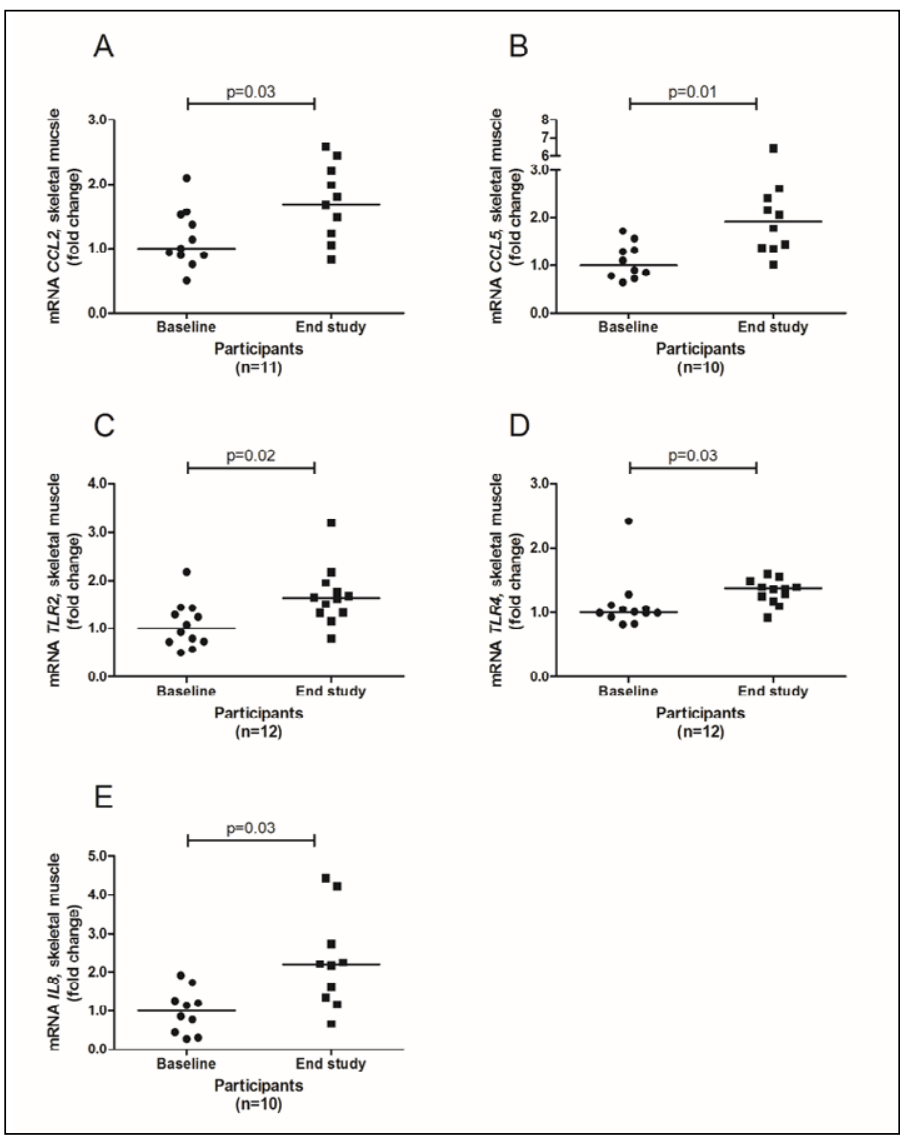

Expression levels of CCL2 [A], CCL5 [B], TLR2 [C], TLR4 [D] and IL8 [E] from baseline to 11 weeks of strength exercise. Values are expressed as fold changes and the vertical lines represent median values. The p-values were calculated by Wilcoxon signed-rank test and indicate changes from baseline til end of study. $\mathrm{n}=$ $10[\mathrm{~B}, \mathrm{E}], \mathrm{n}=11[\mathrm{~A}]$ and $\mathrm{n}=12[\mathrm{C}, \mathrm{D}]$.

It is well known that long-term adaptations to strength training result in increased muscle mass (hypertrophy) and strength (6). Furthermore, regular exercise may reduce the level of immune-related markers (1). This is in line with observations from the present study, as we found reduced mRNA expression levels of IL6, IL8 and CCL3 in PBMCs after the training period. It is also in line with a study where PBMC mRNA expression levels of immune-related markers of middle-aged men and women $(n=40$, mean age 50-67 yrs) were reduced (CCL2), or tended to be reduced (TNF) after two months of brisk walking (6 days/week, $50 \mathrm{~min} /$ day, $70 \%$ of maximal heart rate). However, the mRNA expression level of IL6 did not change after the training period in that study (10). Importantly, the older subjects had higher baseline levels of the relevant markers compared 
to younger participants. The authors therefore suggested that the observed reduction might be related to the higher baseline levels of these mRNA transcripts in the older compared to the younger participants, and that these differences promoted a more robust reduction after the exercise period in older adults (10).

In contrast to the decreased mRNA levels of IL6, IL8 and CCL3 in PBMCs, we observed an increased mRNA expression level of TLR2 in PBMCs as well as in skeletal muscle. The mRNA expression of TLR4, IL8, CCL2 and CCL5 were also significantly increased in skeletal muscle after the intervention. These results are in contrast to others who have found decreased mRNA expression levels of TLRs in PBMCs (19), skeletal muscle (20) and whole blood (21) after regular strength training in older adults. Increased levels of TLRs may induce NF- $\kappa \mathrm{B}$ activation in PBMCs as well as skeletal muscle and contracting $\mathrm{C}_{2} \mathrm{C}_{12}$ myotubes have been shown to induce CCL2 in an NF- $\mathrm{BB}$-dependent manner (22). In skeletal muscle, it has been shown that NF- $\mathrm{BB}$ activation may prevent myogenic differentiation (23) and contribute to muscle atrophy by increasing the activity of molecules involved in muscle protein degradation (24). However, this seems unlikely to occur in the present situation as we observed increased muscle mass after the training period and reduced levels of IL6, IL8 and CCL3 in PBMCs. Similarly, increased NF- $\kappa \mathrm{B}$ activation in PBMCs is closely linked to the development of low-grade inflammation (25), but NF- $\mathrm{BB}$ inhibition during the resolution phase can also prevent proper tissue repair (26). IL8, CCL2 and CCL5 are chemoattractants, which may play an important role in the recruitment of immune-related cells to skeletal muscle following an acute exercise bout (27). Long-term effects of training on these markers are less investigated, but circulating levels of IL8 and CCL2 have been shown to decrease after aerobic training programs (28). The mission of immune-related markers in exercise is not fully understood, but immune-related markers are hypothesized to be important in the resolution processes by removing cellular debris, releasing factors to promote muscle growth and to facilitate vascular and muscle fibre repair, amongst others (29). TLRs may potentially also activate other pathways, such as p38 mitogen-activated kinase (MAPK) and C-Jun N-terminal kinase (JNK) (30) possibly stimulating cell proliferation (31).

Furthermore, we observed decreased mRNA expression levels of NR1H3 in PBMCs, and of PPARGC1A and PPARGC1B in skeletal muscle after the training period. In contrast to these results, regular endurance training has been shown to upregulate the expression of NR1H3 in PBMCs (32) and PPARGC1A and PPARGC1B in skeletal muscle (33). However, a decreased mRNA expression level of PPARGC1B has also been observed in skeletal muscle after 10 weeks of knee extensor training in young adults $(n=7$, mean age $26 \pm 1$ yrs) (34). NR1H3 plays a central role in the transcriptional regulation of both cholesterol homeostasis and inflammation $(32,35)$. The signaling pathway inducing NR1H3 expression in macrophages involves NF- $\kappa \mathrm{B}$ dependent transcriptional gene activation and may promote anti-inflammatory effects (35). Further, TLRs have been shown to downregulate NR1H3 (35), which we also observed in PBMCs in the present study. PPARGC1A and HIF1A are thought to be involved in energy metabolism (36), and PPARGC1A is also closely linked to inflammation (37). Lower levels of PPARGC1A have been observed in patients with diabetes type 2 [38], the metabolic syndrome (39) and in aging (38). A possible explanation for the conflicting results of NR1H3, PPARGC1A and PPARGC1B in the present and other studies may be that participants in the present study also consumed protein supplements, which potentially may alter mRNA expression levels of genes investigated.

\section{Figure 4}

mRNA expression levels in skeletal muscle before and after the intervention

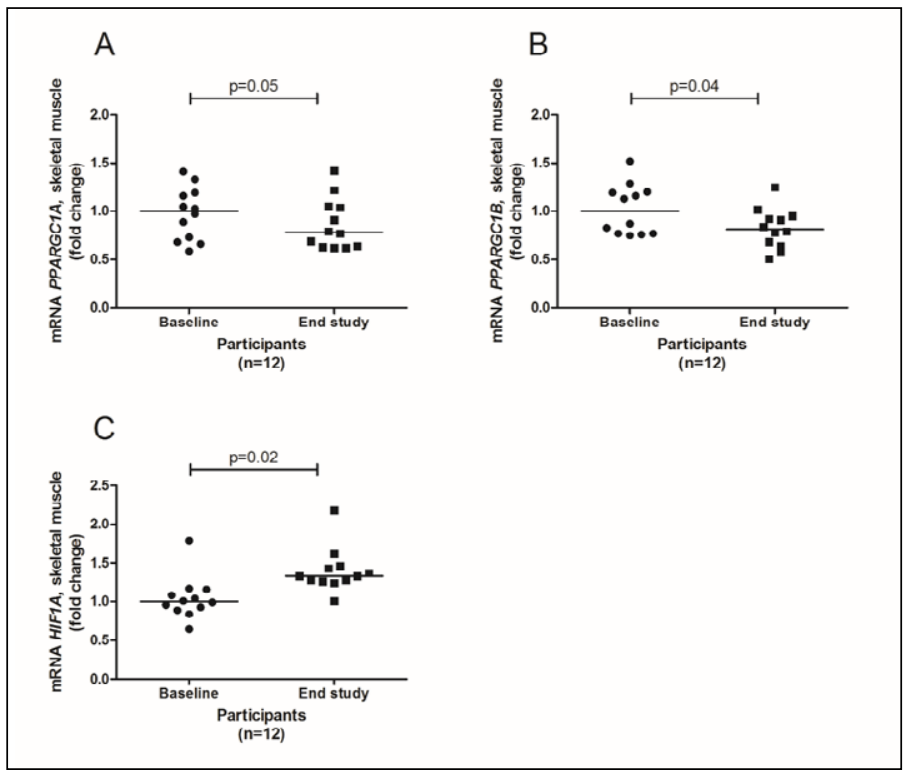

Expression levels of PPARGC1A [A], and PPARGC1B [B] and HIF1A [C] from baseline to 11 weeks of strength exercise. Values are expressed as fold changes and the vertical lines represent median values. The p-values were calculated by Wilcoxon signed-rank test and indicate changes from baseline til end of study. $n$ $=12$.

In contrast to the observed decrease in the mRNA expression level of IL6 in PBMCs in the present study, the circulating level of IL6 was unchanged. This discrepancy support the notion that PBMCs are not the main source of circulating IL6 (40). Further, a stable level of circulating IL6 has previously been found after five consecutive days of high-volume resistance training (41) and after three months of combined endurance and resistance training (42). At the same time, reduced levels of circulating IL6 have been observed after progressive 24-wk exercise of endurance exercise (43) and after walking 10000 steps three times per week for eight weeks (44). Furthermore, we found no changes in the level of CRP after the intervention period. This is supported by a recent study where community dwelling older adults 
performed a strength training program for 12 weeks (45), but is in contrast to the findings in a combined aerobic and resistance training program in middle-aged men and women where the level of CRP was reduced after 6 months of training (46). Strength training has also been shown to decrease CRP in overweight women (47) and in older adults (48).

Possible mechanisms underlying the anti-inflammatory effects of training are largely unknown, but it has been hypothesized that the reduced levels of immunerelated markers observed after training are due to a redistribution of fat mass (1). In the present study, we did not find any significant changes in the distribution of android fat mass and we observed no correlations between changes in mRNA transcripts of IL6, IL8 and CCL3 and android fat mass after the intervention period. Despite no changes in android fat mass, the mRNA expression levels of IL6, IL8 and CCL3 in PBMCs decreased, indicating that the change in PBMCs may occur in the absence of a changed fat distribution.

Protein supplementation, in combination with strength training, may additionally enhance muscle protein synthesis and muscle hypertrophy (49). In addition, whey proteins are hypothesized to exert anti-inflammatory effects (50). CRP was reduced in elderly adults with sarcopenia ( $n=130$, mean age 80.3 yrs) after 12 weeks of supplementation with whey protein $(22 \mathrm{~g})$, essential amino acids ( $10.9 \mathrm{~g}$, including $4 \mathrm{~g}$ leucine) and vitamin $\mathrm{D}(2.5 \mu \mathrm{g})$ concurrent with a combination of regular endurance and strength training (51). Moreover, intake of branched chain amino acids (BCAA) has been shown to attenuate inflammation after a threeday extensive aerobic training program compared to an isocaloric amount of carbohydrate (52). However, no change was observed in serum concentrations of IL6 after resistance training combined with protein and omega-3 supplementation in novice resistance trained females ( $n=28$, mean age $20 \pm 1$ yrs) (41), and a recent metaanalysis showed that whey supplements do not modulate inflammation in healthy adults (53). Nevertheless, in the same meta-analysis, whey supplements lowered serum levels of CRP in subjects with initial high baseline values (53) such as in older adults (9), potentially providing a greater reduction of inflammatory markers in these groups compared to groups with lower baseline levels (54). However, our results do not support this notion, as we observed no differences in circulating levels of CRP or IL6 between the whey and the milk group after the intervention period (results not shown).

Most training studies have been exploring the effects of aerobic exercise on gene expression levels, both in PBMCs and skeletal muscle. Whereas adaptations to endurance training include mitochondrial biogenesis and enhanced aerobic metabolism (55), strength training mainly promotes hypertrophy (56). Different types of exercise are therefore likely to induce different subsets of genes, possibly explaining some of the contradictory observations in the present and previous studies. The fact that subjects in the present study consumed protein supplements combined with the exercise intervention may also have affected mRNA expression levels of selected genes. Further, the differences we observed in mRNA expression levels in PBMCs and in skeletal muscle after eleven weeks of strength training may reflect the different functions and adaptation processes to regular exercise in these two tissues. Differences in initial health status, exercise intensity (57), duration of the exercise performed and sampling points (58) may also explain the conflicting results in PBMCs (16), serum (59) and skeletal muscle (20). The frequency and exercise load may ultimately determine whether the organism responds with favorable adaptations, or, in the case of inadequate recovery, experiences increased inflammation (60). Further, the results from the present study underline the complex regulation and integration of metabolism and inflammation (61) and show that a combined exercise and supplementation intervention were able to alter the expression of genes involved in energy metabolism and inflammation in PBMCs as well as skeletal muscle. The physiological importance of the observed effects of strength training and protein supplementation on immune-related markers in PBMCs, as well as skeletal muscle is unclear and needs further investigations.

Major strengths to the present study are the randomized controlled design, with participants receiving a standardized diet prior to the sampling. All exercise sessions were standardized and performed under close supervision, and blood samples and muscle biopsies were collected simultaneously allowing us to compare the responses in two tissues. There are also some limitations to the present study. Few subjects were included in the study, and the lack of non-significant results may be due to the low number of participants. Further, no adjustments for, or comparison between, gender was performed. We did not include a non-training control group, or a group who did not consume the protein supplements. This makes us unable to conclude that the training intervention was the sole cause of the altered gene expression level observed or to distinguish the effects between the training and the supplementation. The observed changes in some of the inflammatory markers may therefore be related to changes in the diet and may potentially explain the conflicting results between the present intervention study and intervention studies were training is the only intervention.

\section{Conclusion}

In the present study, we found reduced levels of some immune-related markers in PBMCs after eleven weeks of high-load strength training, possibly providing protection against chronically related diseases, such as atherosclerosis. Simultaneously, we observed increased levels of other immune-related mRNA transcripts in PBMCs as well as in skeletal muscle. These changes may 
reflect muscle regeneration and adaptation. However, we need further investigations of the physiological impact of these changes in PBMCs as well as in skeletal muscle. Further, we observed no differences in mRNA expression levels between participants consuming native whey proteins compared to those consuming regular milk proteins during the training period. The present study emphasizes that a combined training and supplementation intervention exert both local (muscle tissue) and systemic effects (PBMCs) and that diet may interfere with this response. However, due to the study design we were not able to separate the effects of the supplementation from the effects of the training.

Acknowledgements: We want to acknowledge all the participants volunteering to the study.

Competing interests: The work was supported by The Research Council of Norway (project number 225258/E40), Throne Holst Foundation for Nutrition Research (University of Oslo), The Norwegian School of Sports Sciences (NIH) and TINE SA.

Conflict of interest: I.O., T.R., H.H., J.J.C., K.B.H. and S.M.U. report no conflict of interest. The test products were provided by TINE SA, Oslo, Norway, where G.O.G. and A.S.B. are researchers employed, G.O.G. as an industrial PhD-student They have no financial interest to declare. K.B.H. has received research grant from TINE SA, Mills DA, Olympic Seafood, Amgen, Sanofi and Pronova. S.M.U. has received research grant from TINE SA, Mills DA and Olympic Seafood. T.R. has received grants from TINE SA.

Ethic approval: The study complies with the current laws in Norway.

Authors' contributions: Conception or design of the study; T.R., H.H., A.S.B. S.M.U. and K.B.H. Acquisition, analysis or interpretation of the work; all authors. Drafting or critically revising the manuscript; all authors. Read and approved the final manuscript; all authors.

\section{References}

1. 1. Gleeson, M., et al., The anti-inflammatory effects of exercise mechanisms and implications for the prevention and treatment of disease. Nat Rev Immunol, 2011. 11(9): p. 607-15.

2. Albers, R., et al., Monitoring immune modulation by nutrition in the general population: identifying and substantiating effects on human health. Br J Nutr, 2013. 110 Suppl 2: p. S1-30.

3. Lee, I.M., et al., Effect of physical inactivity on major non-communicable diseases worldwide: an analysis of burden of disease and life expectancy. Lancet, 2012. 380(9838): p. 219-29.

4. Cruz-Jentoft, A.J., et al., Prevalence of and interventions for sarcopenia in ageing adults: a systematic review. Report of the International Sarcopenia Initiative (EWGSOP and IWGS). Age Ageing, 2014. 43(6): p. 748-59.

5. Lancaster, G.I. and M.A. Febbraio, The immunomodulating role of exercise in metabolic disease. Trends Immunol, 2014. 35(6): p. 262-9.

6. Law, T.D., L.A. Clark, and B.C. Clark, Resistance Exercise to Prevent and Manage Sarcopenia and Dynapenia. Annu Rev Gerontol Geriatr, 2016. 36(1): p. 205-228.

7. Beavers, K.M., et al., Long-term physical activity and inflammatory biomarkers in older adults. Med Sci Sports Exerc, 2010. 42(12): p. 2189-96.

8. Schaap, L.A., et al., Inflammatory markers and loss of muscle mass (sarcopenia) and strength. Am J Med, 2006. 119(6): p. 526.e9-17.

9. Singh, T. and A.B. Newman, Inflammatory markers in population studies of aging. Ageing Res Rev, 2011. 10(3): p. 319-29.

10. Gano, L.B., et al., Increased proinflammatory and oxidant gene expression in circulating mononuclear cells in older adults: amelioration by habitual exercise. Physiol Genomics, 2011. 43(14): p. 895-902.

11. Panagiotakos, D.B., et al., Dairy products consumption is associated with decreased levels of inflammatory markers related to cardiovascular disease in apparently healthy adults: the ATTICA study. J Am Coll Nutr, 2010. 29(4): p. 357-64.

12. Labonte, M.E., et al., Dairy Product Consumption Has No Impact on Biomarkers of Inflammation among Men and Women with Low-Grade Systemic Inflammation. J Nutr, 2014. 144(11): p. 1760-7.

13. Da Silva, M.S. and I. Rudkowska, Dairy nutrients and their effect on inflammatory profile in molecular studies. Mol Nutr Food Res, 2015. 59(7): p. 1249-63.
14. Paulsen, G., et al., Vitamin C and E supplementation alters protein signalling after a strength training session, but not muscle growth during 10 weeks of training. J Physiol, 2014. 592(24): p. 5391-408.

15. Livak, K.J. and T.D. Schmittgen, Analysis of relative gene expression data using real-time quantitative PCR and the 2(-Delta Delta C(T)) Method. Methods, 2001. 25(4): p. 402-8.

16. Gjevestad, G.O., K.B. Holven, and S.M. Ulven, Effects of Exercise on Gene Expression of Inflammatory Markers in Human Peripheral Blood Cells: A Systematic Review. Curr Cardiovasc Risk Rep, 2015. 9(7): p. 34.

17. Paulsen, G., et al., Leucocytes, cytokines and satellite cells: what role do they play in muscle damage and regeneration following eccentric exercise? Exerc Immunol Rev, 2012. 18: p. 42-97.

18. Labonte, M.E., et al., Impact of dairy products on biomarkers of inflammation: a systematic review of randomized controlled nutritional intervention studies in overweight and obese adults. Am J Clin Nutr, 2013. 97(4): p. 706-17.

19. Flynn, M.G., et al., Toll-like receptor 4 and CD14 mRNA expression are lower in resistive exercise-trained elderly women. J Appl Physiol (1985), 2003. 95(5): p. $1833-42$.

20. Greiwe, J.S., et al., Resistance exercise decreases skeletal muscle tumor necrosis factor alpha in frail elderly humans. FASEB J, 2001. 15(2): p. 475-82.

21. McFarlin, B.K., et al., TLR4 is lower in resistance-trained older women and related to inflammatory cytokines. Med Sci Sports Exerc, 2004. 36(11): p. 1876 83

22. Miyatake, S., et al., Contracting C2C12 myotubes release CCL2 in an NF-kappaB-dependent manner to induce monocyte chemoattraction. Am J Physiol Endocrinol Metab, 2016. 310(2): p. E160-70.

23. Langen, R.C., et al., Inflammatory cytokines inhibit myogenic differentiation through activation of nuclear factor-kappaB. Faseb j, 2001. 15(7): p. 1169-80.

24. Li, H., S. Malhotra, and A. Kumar, Nuclear factor-kappa B signaling in skeletal muscle atrophy. J Mol Med (Berl), 2008. 86(10): p. 1113-26.

25. Baker, R.G., M.S. Hayden, and S. Ghosh, NF-kappaB, inflammation, and metabolic disease. Cell Metab, 2011. 13(1): p. 11-22.

26. Lawrence, T., et al., Possible new role for NF-kappaB in the resolution of inflammation. Nat Med, 2001. 7(12): p. 1291-7.

27. Mathers, J.L., et al., Early inflammatory and myogenic responses to resistance exercise in the elderly. Muscle Nerve, 2012. 46(3): p. 407-12.

28. Leggate, M., et al., Determination of inflammatory and prominent proteomic changes in plasma and adipose tissue after high-intensity intermittent training in overweight and obese males. J Appl Physiol (1985), 2012. 112(8): p 1353-60.

29. Smith, C., et al., The inflammatory response to skeletal muscle injury: illuminating complexities. Sports Med, 2008. 38(11): p. 947-69.

30. O'Neill, L.A.J., D. Golenbock, and A.G. Bowie, The history of Toll-like receptors [mdash] redefining innate immunity. Nat Rev Immunol, 2013. 13(6): p. 453-460.

31. Zhang, W. and H.T. Liu, MAPK signal pathways in the regulation of cell proliferation in mammalian cells. Cell Res, 2002. 12(1): p. 9-18.

32. Thomas, A.W., et al., Exercise-associated generation of PPAR ligands activates PPAR signaling events and upregulates genes related to lipid metabolism. Journal of Applied Physiology, 2012. 112(5): p. 806-815.

33. Olesen, J., K. Kiilerich, and H. Pilegaard, PGC-1alpha-mediated adaptations in skeletal muscle. Pflugers Arch, 2010. 460(1): p. 153-62.

34. Mortensen, O.H., et al., PGC-1beta is downregulated by training in human skeletal muscle: no effect of training twice every second day vs. once daily on expression of the PGC-1 family. J Appl Physiol (1985), 2007. 103(5): p. 1536-42.

35. Zelcer, N. and P. Tontonoz, Liver $X$ receptors as integrators of metabolic and inflammatory signaling. J Clin Invest, 2006. 116(3): p. 607-14.

36. Egan, B. and J.R. Zierath, Exercise metabolism and the molecular regulation of skeletal muscle adaptation. Cell Metab, 2013. 17(2): p. 162-84.

37. Handschin, C. and B.M. Spiegelman, The role of exercise and PGC1alpha in inflammation and chronic disease. Nature, 2008. 454(7203): p. 463-9.

38. Ling, C., et al., Multiple environmental and genetic factors influence skeletal muscle PGC-1alpha and PGC-1beta gene expression in twins. J Clin Invest, 2004. 114(10): p. 1518-26.

39. D'Amore, S., et al., Nuclear receptors expression chart in peripheral blood mononuclear cells identifies patients with Metabolic Syndrome. Biochim Biophys Acta, 2013. 1832(12): p. 2289-301.

40. Moldoveanu, A.I., R.J. Shephard, and P.N. Shek, Exercise elevates plasma levels but not gene expression of IL-1beta, IL-6, and TNF-alpha in blood mononuclear cells. J Appl Physiol (1985), 2000. 89(4): p. 1499-504.

41. Hayward, S., et al., Effects of a High Protein and Omega-3-Enriched Diet with or Without Creatine Supplementation on Markers of Soreness and Inflammation During 5 Consecutive Days of High Volume Resistance Exercise in Females. J Sports Sci Med, 2016. 15(4): p. 704-714.

42. Langleite, T.M., et al., Insulin sensitivity, body composition and adipose depots following $12 \mathrm{w}$ combined endurance and strength training in dysglycemic and normoglycemic sedentary men. Arch Physiol Biochem, 2016: p. 1-13.

43. Thompson, D., et al., Time course of changes in inflammatory markers during a 6-mo exercise intervention in sedentary middle-aged men: a randomizedcontrolled trial. J Appl Physiol (1985), 2010. 108(4): p. 769-79. 
44. Yakeu, G., et al., Low-intensity exercise enhances expression of markers of alternative activation in circulating leukocytes: roles of PPARgamma and Th2 cytokines. Atherosclerosis, 2010. 212(2): p. 668-73.

45. Ramel, A., et al., C-Reactive Protein and Resistance Exercise in Community Dwelling Old Adults. J Nutr Health Aging, 2015. 19(7): p. 792-6.

46. Stefanov, T., et al., Effects of supervised vs non-supervised combined aerobic and resistance exercise programme on cardiometabolic risk factors. Cent Eur J Public Health, 2013. 21(1): p. 8-16.

47. Olson, T.P., et al., Changes in inflammatory biomarkers following one-year of moderate resistance training in overweight women. Int J Obes (Lond), 2007. 31(6): p. 996-1003.

48. Ogawa, K., et al., Resistance exercise training-induced muscle hypertrophy was associated with reduction of inflammatory markers in elderly women. Mediators Inflamm, 2010. 2010: p. 171023.

49. Tieland, M., et al., Protein supplementation increases muscle mass gain during prolonged resistance-type exercise training in frail elderly people: a randomized, double-blind, placebo-controlled trial. J Am Med Dir Assoc, 2012. 13(8): p. 713-9.

50. Pal, S. and S. Radavelli-Bagatini, The effects of whey protein on cardiometabolic risk factors. Obes Rev, 2012.

51. Rondanelli, M., et al., Whey protein, amino acids, and vitamin D supplementation with physical activity increases fat-free mass and strength, functionality, and quality of life and decreases inflammation in sarcopenic elderly. Am J Clin Nutr, 2016. 103(3): p. 830-40.
52. Matsumoto, K., et al., Branched-chain amino acid supplementation attenuates muscle soreness, muscle damage and inflammation during an intensive training program. J Sports Med Phys Fitness, 2009. 49(4): p. 424-31.

53. Zhou, L.M., et al., Effect of whey supplementation on circulating C-reactive protein: a meta-analysis of randomized controlled trials. Nutrients, 2015. 7(2): p. 1131-43.

54. Ticinesi, A., et al., Nutrition and Inflammation in Older Individuals: Focus on Vitamin D, n-3 Polyunsaturated Fatty Acids and Whey Proteins. Nutrients, 2016. 8(4): p. 186.

55. Demontis, F., et al., The influence of skeletal muscle on systemic aging and lifespan. Aging Cell, 2013. 12(6): p. 943-9.

56. Ruas, J.L., et al., A PGC-1alpha isoform induced by resistance training regulates skeletal muscle hypertrophy. Cell, 2012. 151(6): p. 1319-31.

57. Izquierdo, M., et al., Cytokine and hormone responses to resistance training. Eur J Appl Physiol, 2009. 107(4): p. 397-409.

58. Suzuki, K., et al., Systemic inflammatory response to exhaustive exercise. Cytokine kinetics. Exerc Immunol Rev, 2002. 8: p. 6-48.

59. Calle, M.C. and M.L. Fernandez, Effects of resistance training on the inflammatory response. Nutr Res Pract, 2010. 4(4): p. 259-69.

60. Kramer, H.F. and L.J. Goodyear, Exercise, MAPK, and NF-kappaB signaling in skeletal muscle. J Appl Physiol (1985), 2007. 103(1): p. 388-95.

61. Hotamisligil, G.S., Inflammation and metabolic disorders. Nature, 2006. 444(7121): p. 860-7 